\title{
Quantum approach to the mechanism of monothiopyrophosphate isomerization
}

\author{
Agata Paneth ${ }^{1} \cdot$ Piotr Paneth $^{2}$ (ID
}

Received: 24 June 2019 / Accepted: 8 August 2019 / Published online: 30 August 2019

(C) The Author(s) 2019

\begin{abstract}
Due to its importance in chemistry and biology, mechanisms of reactions involving phosphates are of central interest. However, only recently, quantum-chemical modeling of these reactions has become possible. With the advent of DFT calculations on phosphate-containing molecules, we have, therefore, investigated theoretically the mechanism of thiol-thione isomerization of a monothiopyrophosphate, which has been for a long time a subject of controversy. The calculations indicate that the reaction proceeds via concerted intramolecular mechanisms.
\end{abstract}

Keywords Thiol-thione isomerization $\cdot$ Thiopyrophosphates $\cdot$ DFT calculations $\cdot$ Isotope effects

\section{Introduction}

Due to the central role of phosphates in biochemistry, mechanisms of reactions of organophosphorus compounds were subjects of intensive studies, with the existence of free metaphosphate being a subject of controversy both in chemistry and in biochemistry for many years [1]. One of the examples of this problem was the mechanism of the thiol-thione rearrangement of monothiophosphate skeleton [2] illustrated by Scheme 1 (atom numbering used throughout the text indicated as subscripts):

Two alternative mechanisms have been proposed as illustrated in Fig. 1. In the upper pathway, the P-S bond dissociates heterolytically to form (intimate) ion pair that recombines via the formation of the P-O bond [3]. In the lower pathway, an internal nucleophilic attack of the oxygen atom on the adjacent phosphorus atom with the formation of a cyclic transition state (or an intermediate) is assumed [4].

This paper belongs to the Topical Collection Zdzislaw Latajka 70th Birthday Festschrift

Piotr Paneth

piotr.paneth@p.lodz.pl

1 Faculty of Pharmacy, Medical University of Lublin, Chodzki 4a, 20-093 Lublin, Poland

2 Faculty of Chemistry, Lodz University of Technology, Zeromskiego 116, 90-924 Lodz, Poland
The problem has been approached with many experimental techniques, one of them being measurements of kinetic isotope effects (KIEs [5]) [6, 7]. We have measured kinetic isotope effects of sulfur [8] and phosphoryl oxygens $[8,9]$ for the reaction carried out in 1-methylnaphthalene, benzonitrile, and propylene carbonate. Based on these measurements and simple modeling, a switch in the mechanism upon change from non-polar to polar solvents has been postulated. However, quantum-chemical modeling of this reaction was then not possible and even with the advent of early ab initio and semiempirical methodology calculations of systems containing phosphorus atoms were scarce and became available only recently.

With the availability of modern density functionals, it is now possible to address the mechanism of the above reaction theoretically. In order to decide on the theory level, a survey of published data has been carried out. Results of older theoretical approaches have been representatively evaluated in the extensive benchmark of calculations on hydrolysis of organophosphorus compounds that has been published in 2010 [10]. Over 50 functionals expressed in Pople's or Dunning's basis sets have been compared with the reference calculations carried out at the CCSD(T)/CBS//B3LYP/6-311++G(2d,2p) theory level. Overall, MPWB1K/6-311+G(2d,2p) calculations [11-14] were found to perform the best. Notably, due to the time when the benchmark was compiled, no functionals that include a correction for dispersion have been evaluated. Since then, an extensive review of theoretical approaches to hydrolysis of biological phosphates has been published recently [15]. 
Scheme 1

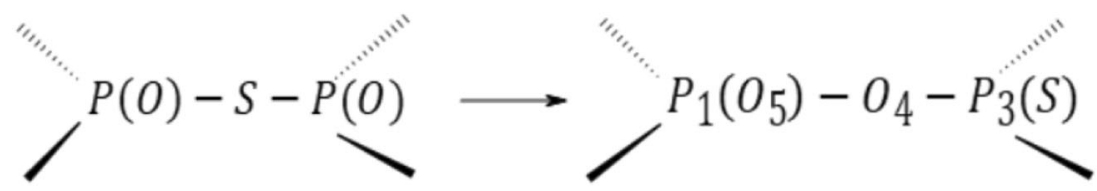

This contribution concentrates, however, on methodological aspects of calculations while employing only SMD/M06-2X/ $6311+\mathrm{G} 9 \mathrm{~d}, \mathrm{p}) / / \mathrm{SMD} / \mathrm{M} 06-2 \mathrm{X} / 6-31+\mathrm{G}(\mathrm{d})$ level of theory. Some other (non-exhaustive) recent examples of using different theory levels in modeling properties and reactivity of phosphoruscontaining molecules include DFTB3/3OB in structure determination [16, 17], B3LYP \& MP3 with 6-311G(d,p) basis set in conformational analysis [18], B3LYP/cc-pVTZ/TIP3P and B3LYP-D3/def2-SVP(D) in studies of anharmonicity of phosphate ions in water $[19,20], \mathrm{M} 06-2 \mathrm{X}, \mathrm{M} 06 \mathrm{~L}$ and PBE with 6$31+\mathrm{G}(\mathrm{d}, \mathrm{p})$ in lipophilicity calculations [21] $\omega \mathrm{B} 97 / 6-311+$ $\mathrm{G}(3 \mathrm{df}, 2 \mathrm{p}) / / \mathrm{M} 06-2 \mathrm{X} / 6-311+\mathrm{G}(\mathrm{d}, \mathrm{p})$ in investigations of the reaction with $\mathrm{OH}$ radical [22], MP3/6-311++G(2d,2p)//B3LYP/6 $-31+\mathrm{G}(\mathrm{d})$ in hydrolysis of pesticides [23], PM3/MM, BLYP/631G(d)/MM, B3LYP/6-31G*/MM and COSMO/B3LYP/6$31 \mathrm{G}(\mathrm{d})$ in hydrolysis of monoesters [24], B3LYP/6-311+ $\mathrm{G}(\mathrm{d}, \mathrm{p})$ and $\mathrm{M} 06-2 \mathrm{X} / 6-311+\mathrm{G}(\mathrm{d}, \mathrm{p})$ in gas-phase decomposition [25], B3LYP/6-311+G(d,p) in metathiophosphate di- and polymerization [26], M06-2X/6-311+G(3df,2p) and B2PLYP/ $6311+\mathrm{G}(3 \mathrm{df}, 2 \mathrm{p}) / /(\mathrm{IEFPCM}) / \mathrm{M} 06-2 \mathrm{X} / 6-311+\mathrm{G}(\mathrm{d}, \mathrm{p})$ in oxidation [27], PBE-D3/PAW in non-aqueous degradation [28], M06-2X/6-31G(d,p) and BLYP-D3/aug-cc-pVTZ in binding to an enzyme [29], and B97D/6-31+G(d)/AMBER [30], B3LYP/6-31G(d) [31] and PBE0-D3/def2-TZVP [32] in enzymatic catalysis.

In this contribution, we have approached modeling of the alternative pathways of the isomerization of bis(5,5-dimethyl- 2-oxo-1,2,4-dioxaphosphorinanyl) sulfide using contemporary computational tools. Based on the examples given above, a density functional augmented by dispersion correction, namely $\omega \mathrm{B} 97 \mathrm{xD}$ [33], expressed in a triple-zeta, 6-311++ G(d) [34], basis set has been chosen. This theory level, augmented by continuum solvent model, allowed for analysis of both alternative pathways and associated kinetic isotope effects.

\section{Methods}

All calculations were performed using Gaussian16 package [35] and visualized with GaussView6 program [36]. All calculations were first carried out using $\omega \mathrm{B} 97 \mathrm{xD}$ functional expressed in the def2-SVPP basis set [37]. Harmonic frequencies were calculated to validate optimization to stationary points. IRC protocol [38] was employed for the confirmation that the transition states corresponded to studied reactions. The solvent was represented by the PCM model [39]. The reaction in 1-methylnaphthalene has been modeled in the gas phase, and using parameters for $\mathrm{p}$-xylene. For the reaction in benzonitrile, the parameters for this solvent were employed. Since, however, parameters for propylene carbonate are not available, we have used these for water, which provides the high extreme on the polarity scale to ensure the polarity of the solvent larger than that under experimental conditions.
Fig. 1 Assumed alternative mechanisms of organic monothiophosphate isomerization (see text for details)
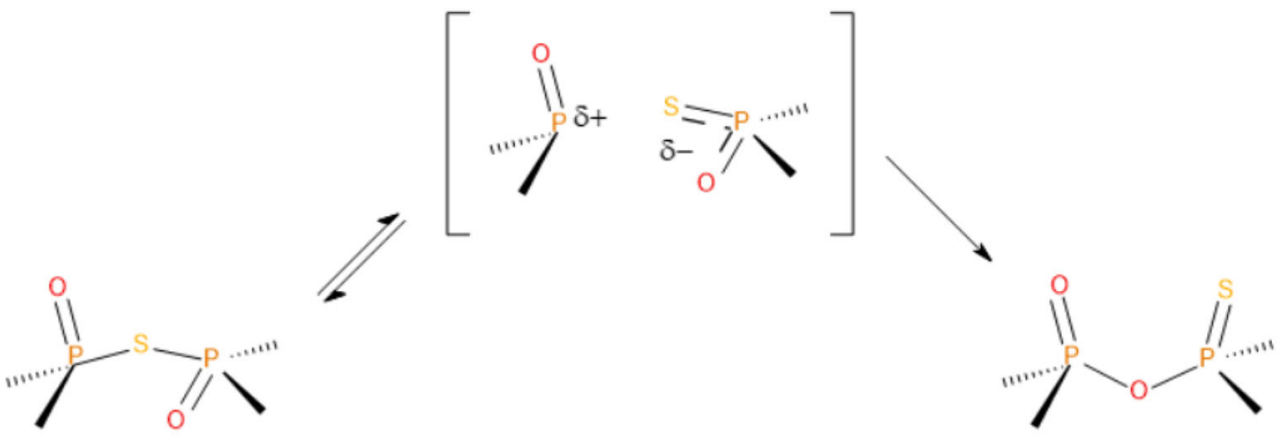

1

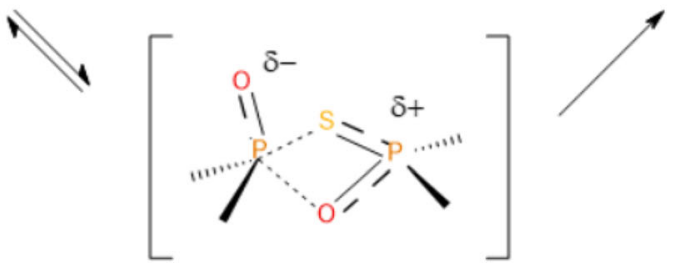

2 
Table 1 Partial atomic charges (a.u.) on exocyclic oxygen atom and sulfur atom in 5,5-dimethyl-1,3,2-dioxaphosphorinane-2-oxo-2-thio anion calculated with $\omega \mathrm{B} 97 \mathrm{xD}$ functional and different basis sets

\begin{tabular}{lllr}
\hline Basis set & $\delta$ on oxygen & $\delta$ on sulfur & $\delta \mathrm{S}-\delta \mathrm{O}$ \\
\hline def2-SVPP & -0.622 & -0.658 & -0.04 \\
def2-TZVP & -0.571 & -0.649 & -0.08 \\
def2-QZVP & -0.707 & -0.686 & -0.02 \\
6-31+G & -0.884 & -0.433 & 0.45 \\
6-31+G(d) & -0.888 & -0.595 & 0.29 \\
6-31+G(d,p) & -0.889 & -0.595 & 0.29 \\
$6-31++G$ & -0.855 & -0.413 & 0.44 \\
6-311+G & -0.456 & -0.698 & -0.24 \\
6-311+G(d) & -0.491 & -0.648 & -0.16 \\
6-311++G & -0.404 & -0.728 & -0.33 \\
6-311++G(d) & -0.447 & -0.667 & -0.22 \\
6-311++G(d,p) & -0.446 & -0.671 & -0.22 \\
aug-CC-pVDZ & -0.992 & -0.780 & 0.21 \\
aug-CC-pVTZ & -0.966 & -0.953 & 0.20 \\
aug-CC-pVQZ & -1.320 & -0.691 & 0.63 \\
\hline
\end{tabular}

Subsequently, stationary points were re-optimized at the $\mathrm{PCM}(\mathrm{aq}) / \omega \mathrm{B} 97 \mathrm{xD} / 6-311++\mathrm{G}(\mathrm{d})$ level of theory and harmonic frequencies obtained at this theory level were used in calculations of isotope effects by means of our own Isoeff2017 program [40].

\section{Results and discussion}

Selection of the theory level Two trends can be seen from the examples discussed in the Introduction; firstly, there is a shift from the robust B3LYP functional $[41,42]$ to those that take into account dispersion, and secondly, the majority of calculations have been performed with a triple-zeta basis set. Our failures in finding the transition state for the dissociative pathway at the B3LYP/6-31+G(d) and $\omega \mathrm{B} 97 \mathrm{xD} / \mathrm{def} 2-\mathrm{SVPP}$ levels of theory are in line with the observation that the basis set of the triple-zeta quality is necessary for reactions that involve reactions at the phosphorus center. We have, therefore, decided to employ $\omega \mathrm{B} 97 \mathrm{xD}$ functional, which we found performing very well for other systems [43]. The decision on the basis set has been made on the basis of charge distribution in phosphathioate anion that we have studied experimentally. Our ${ }^{31} \mathrm{P}-\mathrm{NMR}$ results indicate that there is little charge delocalization in this anion with the extra electron localized mostly on the sulfur atom [44]. Many theory levels and charge partitioning schemes fail to correctly distribute electron density in agreement with this observation [45]. We have, therefore, evaluated a number of basis sets taking into consideration Mülliken partial atomic charges on sulfur and exocyclic oxygen. The results are collected in Table 1 . The results obtained with the 6-311++G basis set are in the best agreement with the experimental observation while the increase of polarization of the basis set leads to opposite charge distribution. Since our goal has been studies of reaction mechanisms, the polarization functions needed to be employed. We have, therefore, resorted to the 6-311++G(d) basis set. APT partial atomic charges, while generally larger in absolute values, led qualitatively to the same results (data not shown). Furthermore, in order to facilitate calculations, we have decided to use "cheaper" Ahlrichs def2-SVPP basis set that preserves the trend of this charge distribution, for initial scans, IRCs, and optimizations and to resort to $\omega \mathrm{B} 97 \times \mathrm{xD} / 6-311++\mathrm{G}(\mathrm{d})$ level only for final calculations.

Isomerization mechanisms We have identified three pathways leading from the symmetrical thiopyrophosphate $\mathbf{1}$ to the asymmetrical product $\mathbf{2}$. The corresponding transition state structures are illustrated in Fig. 2 and the main structural data collected in Table 2.

Reported in Table 2, bond orders were obtained from the Pauling equation: [46].

$r_{n}=r_{1}-c \cdot \ln (\mathrm{n})$

where $r$ is the bond length and $n$ is the bond order. Indices $n$ and 1 correspond to $n$-order and single bond, respectively. Coefficients $c$ for P-S and P-O bonds were evaluated on the

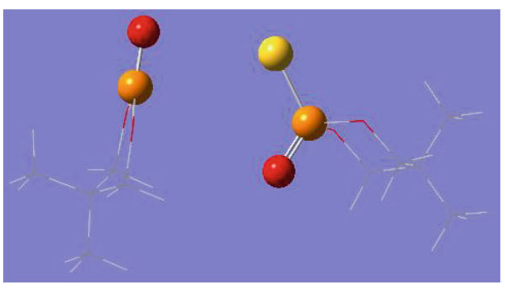

TSd

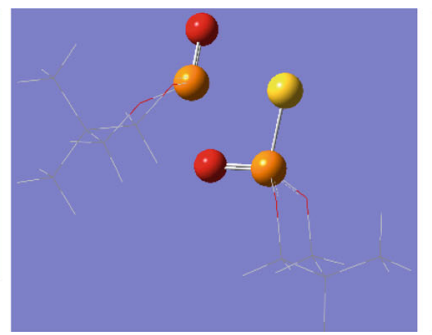

TSi1

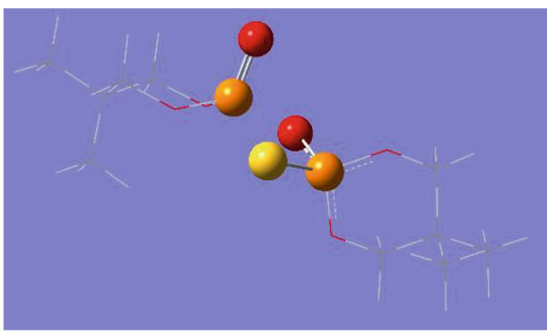

TSi2

Fig. 2 Transition state structures obtained for the dissociative mechanism (left) and two associative mechanisms (middle and right). For clarity, parts remote to the reaction center are rendered as wireframes 
Table 2 Main geometric and energetic parameters ( $\mathrm{kcal} / \mathrm{mol})$ of the reactants and transition state structures at the $\omega \mathrm{B} 97 \mathrm{xD} / 6-311++\mathrm{G}(\mathrm{d}) / \mathrm{PCM}(\mathrm{aq})$ level of theory. Atom numbering follows the Scheme in the Introduction

\begin{tabular}{|c|c|c|c|c|c|}
\hline Parameter & 1 & TSd & TSi1 & TSi2 & 2 \\
\hline \multicolumn{6}{|c|}{ Interatomic distances (in $\AA$ ) } \\
\hline $\mathrm{P}_{1}-\mathrm{O}_{5}$ & 1.477 & 1.443 & 1.486 & 1.486 & 1.471 \\
\hline $\mathrm{P}_{1}-\mathrm{S}$ & 2.091 & 4.487 & 2.411 & 2.417 & 4.536 \\
\hline $\mathrm{S}-\mathrm{P}_{3}$ & 2.112 & 1.979 & 1.994 & 1.983 & 1.924 \\
\hline $\mathrm{P}_{3}-\mathrm{O}_{4}$ & 1.474 & 1.503 & 1.530 & 1.560 & 1.604 \\
\hline $\mathrm{O}_{4}-\mathrm{P}_{1}$ & 3.724 & 4.836 & 2.131 & 1.844 & 1.613 \\
\hline \multicolumn{6}{|c|}{ Dihedral angles (in degrees) } \\
\hline $\mathrm{P}_{1}-\mathrm{S}-\mathrm{P}_{3}-\mathrm{O}_{4}$ & -56.6 & -32.0 & 4.9 & -4.6 & 30.6 \\
\hline $\mathrm{O}_{5}-\mathrm{P}_{1}-\mathrm{P}_{3}-\mathrm{S}$ & 128.3 & -15.6 & 35.9 & 99.3 & 167.4 \\
\hline \multicolumn{6}{|c|}{ Pauling bond orders } \\
\hline $\mathrm{P}_{1}-\mathrm{O}_{5}$ & 1.97 & 2.35 & 1.88 & 1.88 & 2.03 \\
\hline $\mathrm{P}_{1}-\mathrm{S}$ & 1.04 & 0.00 & 0.30 & 0.29 & 0.00 \\
\hline $\mathrm{S}-\mathrm{P}_{3}$ & 0.96 & 1.61 & 1.52 & 1.59 & 2.00 \\
\hline $\mathrm{P}_{3}-\mathrm{O}_{4}$ & 2.00 & 1.72 & 1.50 & 1.28 & 1.02 \\
\hline $\mathrm{O}_{4}-\mathrm{P}_{1}$ & 0.00 & 0.00 & 0.07 & 0.30 & 0.98 \\
\hline \multicolumn{6}{|c|}{ Partial atomic charges } \\
\hline $\mathrm{O}_{5}$ & -0.7 & -0.03 & 0.12 & -0.26 & -0.11 \\
\hline $\mathrm{S}$ & -0.11 & -0.66 & -0.31 & -0.12 & -0.38 \\
\hline $\mathrm{O}_{4}$ & 0.01 & -0.44 & -0.47 & -0.23 & 0.00 \\
\hline $\mathrm{SP} 3(\mathrm{O} 4)^{/}$fragment & -0.12 & -0.96 & -0.08 & 0.62 & -0.09 \\
\hline \multicolumn{6}{|c|}{ Energetics (in kcal/mol) } \\
\hline$\Delta \mathrm{G}^{\neq}$or $\Delta \mathrm{G}_{\mathrm{r}}$ & 0 & 52.1 & 32.7 & 29.3 & -10.2 \\
\hline
\end{tabular}

basis of averaged bond lengths for single and double bonds in reactants.

The three localized transition state structures are characterized by different charge distribution; in the TSd structure, practically full charge separation between dissociating fragments is observed. The breaking $\mathrm{P}_{1}-\mathrm{S}$ distance is nearly $4.5 \AA$. However, from the last line of Table 2, it is apparent that the dissociative mechanism can be excluded from further considerations as the formation of the ion pair that recombines to the product is energetically too demanding. The TSi1 transition state structure shows moderate $\mathrm{P}_{1}-\mathrm{S}$ bond breaking that is concertedly compensated by the significant shortening of the $\mathrm{P}_{1}-\mathrm{O}_{4}$ distance. The charge distribution between the two fragments stays, however, neutral as in both reactants. Transition state structure TSi2 resembles geometrically TSi1, with main distinctions being a rotation of the $\mathrm{P}_{1} \mathrm{SP}_{3} \mathrm{O}_{4}$

Table 3 Calculated and experimental kinetic isotope effects at $404 \mathrm{~K}$

\begin{tabular}{lllll}
\hline Isotope effect & TSd & TSi1 & TSi2 & Experiment \\
\hline $\mathrm{k}_{16} / \mathrm{k}_{18}$ & 1.0046 & 1.0154 & 1.0062 & $1.0026 \pm 0.0009[8]$ \\
$\mathrm{k}_{32} / \mathrm{k}_{36}$ & 1.0286 & 1.0150 & 1.0223 & $1.0244 \pm 0.0009[9]$ \\
\hline
\end{tabular}

plane by about $90^{\circ}$ and the formation of the $\mathrm{P}_{1}-\mathrm{O}_{4}$ bond being even more advanced. The charge on the SP3(O4)/ fragment is positive in accordance with the synchronous breaking of the $\mathrm{P}_{3}-\mathrm{S}$ bond and formation of the $\mathrm{P}_{1}-\mathrm{O}_{4}$ bond and building the negative charge on the $\mathrm{O}_{5}$, although surprisingly, this is not paralleled by the elongation of the $\mathrm{P}_{1}-\mathrm{O}_{5}$ bond. Attempts to localize a cyclic intermediate failed at all considered theory levels indicating that such structure is not a stationary point. The concerted mechanism proceeding via TSi2 requires less energy than the one characterized by the TSi1. In fact, the calculated Gibbs free energy of activation of $29.3 \mathrm{kcal} / \mathrm{mol}$ is in excellent agreement with the experimentally determined enthalpy of activation of $29.9 \pm 1.3 \mathrm{kcal} / \mathrm{mol}$ [47].

Table 4 Dependence of the calculated sulfur KIE for the reaction proceeding via TSi2 on the polarity of the solvent at $\omega \mathrm{B} 97 \mathrm{xD} / \operatorname{def} 2-\mathrm{SVPP} /$ PCM theory level

\begin{tabular}{ll}
\hline SCRF & $\begin{array}{l}\mathrm{k}_{32} / \\
\mathrm{k}_{36}\end{array}$ \\
\hline- & 1.0219 \\
p-Xylene & 1.0228 \\
Benzonitrile & 1.0230 \\
Water & 1.0240 \\
\hline
\end{tabular}


Scheme 2

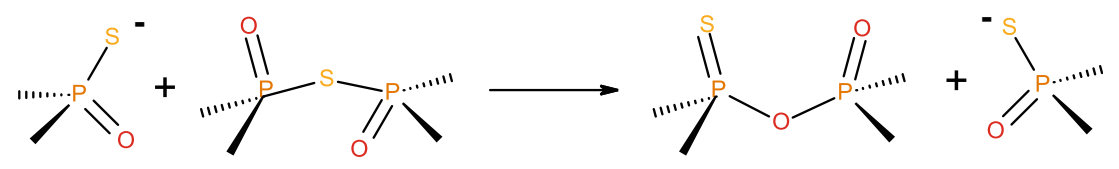

Isotope effects Isotope effects for reaction channels proceeding via all three transition states are collected in Table 3. For direct comparison with the experimental data, we have calculated isotope effects of sulfur-36, and oxygen-18 averaged over both exocyclic positions.

As can be seen, kinetic isotope effects obtained from TSi1 do not agree with the experimental data; the oxygen KIE is substantially larger while sulfur KIE substantially smaller than values obtained experimentally. The results obtained for the pathway proceeding via TSi 2 on the other hand agree very well with the experimental data. We thus conclude that the reaction proceeds via cyclic albeit non-synchronous transition state: the P-S bond breaking is advanced by some $70 \%$ while the formation of the new P-O bond is advanced only to some $30 \%$ (taking into account bond orders).

The above findings leave unsolved one observation, namely the decrease of sulfur KIE in the polar solvent; $\mathrm{k}_{32} / \mathrm{k}_{36}$, in propylene carbonate, has been found equal to $1.0210 \pm 0.0014$. Our calculations indicate that the increase of polarity of the solvent leads to an increase of the isotope effect, as illustrated in Table 4, which does not agree with this experimental observation.

Alternative mechanisms In the search for the explanation of this discrepancy, we have considered a few alternative mechanisms suggested in the literature. The possibility of bimolecular reaction between two molecules of monothiopyrophosphate [48] has been excluded since it is hard even to construct a model of reactants due to steric effects. It is, however, known that it is very hard to obtain and store propylene carbonate without traces of water. Thus, another alternative mechanistic option that has been considered is a reaction between phosphathioate anion and symmetrical reactant illustrated by Scheme 2:

Small amounts of the anion could result from tracer amounts of water in propylene carbonate which can hydrolyze the reactant 1 . In fact, the energetic cost of this hydrolysis has been found to be about $16.5 \mathrm{kcal} / \mathrm{mol}$ when hydroxyl ion is considered as the nucleophile. The ${ }^{36} \mathrm{~S}-\mathrm{KIE}$ for this reaction is small (1.0065). The reaction between phosphathioate anion and reactant $\mathbf{1}$ is also feasible under studied conditions; calculated Gibbs free energy of activation equals to $25.7 \mathrm{kcal} / \mathrm{mol}$. Again, sulfur KIEs for this reaction are much smaller than the isotope effect calculated for the unimolecular reaction (1.0097 for the sulfur atom in the leaving group and 0.9989 for the sulfur atom in the incoming group). Thus, small participation of this reaction channel in the formation of the product 2 can explain the observed decrease of the sulfur isotope effect.

\section{Conclusions}

A few general conclusions arise from the studies presented herein. The most important is the mechanistic finding that, opposite to previous interpretation, the mechanism of unimolecular thiol-thione isomerization of organic monothiopyrophosphates proceed via a cyclic transition state with the P-S bond breaking much more advanced than the formation of the new P-O bond. The lower value of the sulfur kinetic isotope effect observed in a polar solvent, propylene carbonate, most probably results from the participation of side bimolecular reaction of the reactant with phosphathiolate anion and is not the result of the change in the mechanism of the unimolecular reaction. It should be kept in mind that continuum solvent models neglect direct interactions of solvent molecules with the reactants. Due to molecular size of all solvents of interest, however, studies with explicit solvation are prohibitively time-consuming. We have, therefore, studied possibility of direct interactions between ionic fragments formed in the dissociative pathway and a single molecule of benzonitrile or propylene carbonate (data not shown) and found them to be energetically disfavored. In the studied system, interactions of 1-methylnaphtalene with reactants is rather unlikely.

A somewhat surprising observation has been made that atomic partial charge localization does not correlate directly with bond lengths. More studies are required to investigate this phenomenon in terms of the theory level used, especially the basis set, for other phosphorus-sulfurand phosphorus-oxygen-containing organophosphorus compounds.

Results obtained using cheaper B3LYP/6-31+G(d) and $\omega \mathrm{B} 97 \mathrm{xD} / \mathrm{Def} 2-\mathrm{SVPP}$ theory levels are very similar to those obtained with $\omega \mathrm{B} 97 \times \mathrm{D} / 6-311++\mathrm{G}(\mathrm{d})$ for energetics and kinetic isotope effects. However, only at the triple-zeta level of the basis set it was possible to localize the transition state for the dissociative pathway.

Funding information Computational grant at Cyfronet, Cracow, within the PL-Grid network is gratefully acknowledged.

Open Access This article is distributed under the terms of the Creative Commons Attribution 4.0 International License (http:// creativecommons.org/licenses/by/4.0/), which permits unrestricted use, distribution, and reproduction in any medium, provided you give appropriate credit to the original author(s) and the source, provide a link to the Creative Commons license, and indicate if changes were made. 


\section{References}

1. Westheimer FH (1987) Why nature chose phosphates. Science 235: 1173-1178

2. Michalski J, Reimschüssel W, Kamiński R, Paneth P (1987) Mechanism of isomerization of sym-monothiopyrophosphates. Phosphorus and Sulfur 30:257-260

3. Michalski J, Ann NY (1972) The chemistry and stereochemistry of organic selenium-phosphorus acids and phosphine selenides. Acad Sci 192:90-100

4. Michalski J (1963) Études dans le domaine des dérivé organiques de quelques pyroacides du phosphore. Bull Soc Chim Fr 11

5. Wolfsberg M, Van Hook A, Paneth P (2010) Isotope effects in the chemical, geological and bio sciences. Springer, London

6. Hengge AC, Cleland WW (1990) Direct measurement of transitionstate bond cleavage in hydrolysis of phosphate esters of p-nitrophenol. J Am Chem Soc:7421-7422

7. Hengge AC (2002) Isotope effects in the study of phosphoryl and sulfuryl transfer reactions. Acc Chem Res 35:105-112

8. Paneth P (1983) Kinetic isotope effects of oxygen and sulphur on monothiopyrophosphate isomerization on the example of bis(5,5dimethyl-2-oxo-1,3,2-dioxaphosphorinanyl) sulfide (in Polish) dissertation, Lodz University of Technology, Lodz

9. Paneth P, Reimschüssel W (1985) Relative ${ }^{36} \mathrm{~S} /{ }^{34} \mathrm{~S}$ kinetic isotope effects. J Am Chem Soc 107:1407-1408

10. Ribeiro AJM, Ramos MJ, Fernandes PA (2010) Benchmarking of DFT functionals for the hydrolysis of phosphodiester bonds. J Chem Theory Comput 6:2281-2292

11. Becke AD (1996) Density-functional thermochemistry 0.4. A new dynamical correlation functional and implications for exactexchange mixing. J Chem Phys 104:1040-1046

12. Zhao Y, Truhlar DG (2004) Hybrid meta density functional theory methods for thermochemistry, thermochemical kinetics, and noncovalent interactions: the MPW1B95 and MPWB1K models and comparative assessments for hydrogen bonding and van der Waals interactions. J Phys Chem A 108:6908-6918

13. Perdew JP (1991) Unified theory of exchange and correlation beyond the local density aproximation. In: Zieche P, Eschrig H (eds) Electronic structure of solids. Germany, Berlin, pp 11-20

14. Adamo C, Barone V (1998) Exchange functionals with improved long-range behavior and adiabatic connection methods without adjustable parameters: the mPW and mPW1PW models. J Chem Phys 108:664-675

15. Petrovič D, Szeler K, Kamerlin SCL (2018) Challenges and advances in the computational modeling of biological phosphate hydrolysis. Chem Commun 54:3077-3089

16. Yang Y, Yu H, York D, Elstner M, Cui Q (2008) Description of phosphate hydrolysis reactions with the self-consistent-charge density-functional-tight-binding (SCC-DFTB) theory. 1. Parameterization. J Chem Theory Comput 4:2067-2084

17. Gaus M, Lu X, Marcus Elstner M, Qiang Cui Q (2014) Parameterization of DFTB3/3OB for sulfur and phosphorus for chemical and biological applications. J Chem Theory Comput 10: $1518-1537$

18. Sahu C, Das AK (2017) Solvolysis of organophosphorus pesticide parathion with simple and $\alpha$ nucleophiles: a theoretical study. J Chem Sci 129:1301-1317

19. Mendieta-Moreno JI, Walker RC, Lewis JP et al (2019) Fireball/ amber: an efficient local-orbital DFT QM/MM method for biomolecular systems. J Chem Theory Comput 15:1924-1938

20. Bikramjit S, Amalendu C (2017) Ab initio molecular dynamics simulation of the phosphate ion in water: insights into solvation shell structure, dynamics, and kosmotropic activity. J Phys Chem B 121:10519-10529
21. Vlahović F, Ivanović S, Zlatar M, Gruden M (2017) Density functional theory calculation of lipophilicity for organophosphate type pesticides. J Serb Chem Soc 82:1369-1378

22. Li C, Zheng S, Chen J, Xie H-B, Zhang Y-N, Zhao Y, Du Z (2018). Chemosphere 201:557-563

23. Dyguda-Kazimierowicz E, Roszak S, Sokalski WA (2014) Alkaline hydrolysis of organophosphorus pesticides: the dependence of the reaction mechanism on the incoming group conformation. J Phys Chem B 118:7277-7289

24. Plotnikov NV, Prasad BR, Suman Chakrabarty S et al (2013) Quantifying the mechanism of phosphate monoester hydrolysis in aqueous solution by evaluating the relevant ab initio QM/MM free energy surfaces. J Phys Chem B 117:12807-12819

25. Jiang K, Zhang N, Zhang H et al (2015) Investigation on the gasphase decomposition of trichlorfon by GC-MS and theoretical calculation. PLoS One 10:e121389

26. Mosey NJ, Woo TK (2006) An ab initio molecular dynamics and density functional theory study of the formation of phosphate chains from metathiophosphates. Inorg Chem 45:7464 7479

27. Chao L, Jingwen C, Hong-Bin X et al (2017) Effects of atmospheric water on $\mathrm{OH}$-initiated oxidation of organophosphate flame retardants: a DFT investigation on TCPP. Environ Sci Technol 51: 5043-5051

28. Gallis DFS, Harvey JA, Pearce CJ et al (2018) Efficient MOFbased degradation of organophosphorus compounds in nonaqueous environments. J Mater Chem A 6:3038-3045

29. Allgardssona A, Bergb L, Akfura C et al (2016) Structure of a prereaction complex between the nerve agent sarin, its biological target acetylcholinesterase, and the antidote HI-6. PNAS 113:55145519

30. McClory J, Hu G-H, Zou J-W et al (2019) Phosphorylation mechanism of $\mathrm{N}$-acetyl L-glutamate kinase, a QM/MM study. J Phys Chem B 123:2844-2852

31. Maslova O, Aslanli A, Stepanov N et al (2017) Catalytic characteristics of new antibacterials based on hexahistidine-containing organophosphoris hydrolase. Catalyst 7:271

32. Zlobin A, Mokrushina Y, Terekhov S et al (2018) QM/MM description of newly selected catalytic bioscavengers against organophosphorus compounds revealed reactivation stimulus mediated by histidine residue in the acyl-binding loop. Front Pharmacol 9:834

33. Chai JD, Head-Gordon M (2008) Long-range corrected hybrid density functionals with damped atom-atom dispersion corrections. Phys Chem Chem Phys 10:6615-6620

34. McLean AD, Chandler GS (1980) Contracted Gaussian-basis sets for molecular calculations. 1. 2nd row atoms, $Z=11-18$. J Chem Phys 72:5639-5648

35. Frisch M J, Trucks G W, Schlegel H B et al. (2016) Gaussian, Version 16, Revision A.03; Gaussian, Inc.: Wallingford, CT, USA

36. Gauss View, version 6; Gaussian, Inc.: Wallingford, CT, USA, 2016

37. Weigend F, Ahlrichs R (2005) Balanced basis sets of split valence, triple zeta valence and quadruple zeta valence quality for $\mathrm{H}$ to $\mathrm{Rn}$ : design and assessment of accuracy. Phys Chem Chem Phys 7: 3297-3305

38. Fukui K (1981) The path of chemical-reactions - the IRC approach. Acc Chem Res 14:363-368

39. Miertuš S, Scrocco E, Tomasi J (1981) Electrostatic interaction of a solute with a continuum. A direct utilization of ab initio molecular potentials for the prevision of solvent effects. Chem Phys 55:117129

40. Anisimov V, Paneth P (1999) ISOEFF98. A program for studies of isotope effects using hessian modifications. J Math Chem 26:75-86

41. Becke AD (1993) Density-functional thermochemistry. III. The role of exact exchange. J Chem Phys 98:5648-5652 
42. Lee C, Yang W, Parr RG (1988) Development of the colle-salvetti correlation-energy formula into a functional of the electron density. Phys Rev B 37:785-789

43. Pokora M, Paneth P (2018) Can adsorption on graphene be used for isotopic enrichment? A DFT perspective. Molecules 23:2981

44. Frey PA, Reimschüssel W, Paneth P (1986) Phosphorus-sulfur bond order in phosphothiate anions. J Am Chem Soc 108:1720-1722

45. Rostkowski M, Paneth P (2007) Charge localization in monothiophosphate monoanions. Pol J Chem 81:711-720

46. Pauling L (1947) Atomic radii and interatomic distances in metals. J Am Chem Soc 69:542-553
47. Kamiński R (1978) Mechanims of isomerization of organic monothopyrophosphates on the example of bis(5,5-dimethyl-2oxo-1,3,2-dioxaphosphorinanyl) sulfide" (in Polish) dissertation, Centre of Molecular and Macromolecular Studies, Polish Academy of Sciences, Lodz

48. Młotkowska B (1969) Studies of synthesis and chemical reactions of monothiopyrophosphates (in Polish) dissertation. Lodz University of Technology, Lodz

Publisher's note Springer Nature remains neutral with regard to jurisdictional claims in published maps and institutional affiliations. 